\title{
Influencia de la religiosidad, la norma subjetiva y la efectividad percibida del consumidor en el consumo socialmente responsable *
}

\author{
Influence of Religiosity, Subjective Norm, and Perceived Consumer \\ Effectiveness on Socially Responsible Consumption
}

\author{
Juan Camilo Mejía \\ Doctorante en Marketing, Universidad de Valencia, \\ Valencia-España,mejuanca@alumni.uv.es \\ Rafael Currás-Pérez \\ Doctor en Marketing, Universidad de Valencia, \\ Valencia-España, rafael.curras-perez@uv.es \\ Carlos Manuel Córdoba-Segovia \\ Doctor en Globalización Económica y Sociedad de la Información, Universidad de Nariño, \\ San Juan de Pasto-Colombia, carcord13@udenar.edu.co
}

Cómo citar / How to cite

Mejía, J. C.; Currás-Pérez, R.; Córdoba-Segovia, C. M. (2021). Influencia de la religiosidad, la norma subjetiva y la efectividad percibida del consumidor en el consumo socialmente responsable. Revista CEA, v. 7, n. 14, e1809. https://doi.org/10.22430/24223182.1809

Recibido: 25 de enero de 2021

Aceptado: 19 de abril de 2021

\begin{abstract}
Resumen
Esta investigación analizó la relación entre religiosidad, norma subjetiva y efectividad percibida del consumidor en la intención de compra de productos socialmente responsables. La investigación empírica se basó en un cuestionario repartido, en igual proporción, a 485 personas ubicadas en Colombia y España. Para su evaluación se realizó un modelo de ecuaciones estructurales a partir de la técnica Partial Least Square (PLS). Se encontró similitud en los resultados entre ambos países, comprobándose, en los dos casos, la influencia de la religiosidad sobre las dimensiones de consumo
\end{abstract}

* Este artículo se deriva del proyecto titulado «Influencia de la religiosidad, la norma subjetiva y la efectividad percibida del consumidor en el consumo socialmente responsable» y ha sido financiado con recursos propios. 
Influencia de la religiosidad, la norma subjetiva y la efectividad percibida del consumidor en el consumo socialmente responsable

responsable. También se pudo determinar la importancia de la norma subjetiva sobre este tipo de consumo. Sin embargo, no fue posible comprobar la hipótesis que relacionaba la efectividad percibida por el consumidor en este tipo de compras. Con estos resultados se concluyó acerca de la importancia del papel de la religiosidad en las intenciones de consumo responsable, debido, principalmente, a su función simbólica y socializadora como elemento de aculturación y de expresión de identidad. Asimismo, fue posible ampliar la teoría del comportamiento planificado al explorar la norma subjetiva en este tipo de compras. El estudio sugirió investigaciones sobre consumo responsable y sus factores influyentes, y propuso estrategias de marketing para fortalecerlo en favor del medio ambiente, la sociedad y la economía de las organizaciones.

Palabras clave: estructura del mercado, comportamiento del mercado, efectividad percibida del consumidor, norma subjetiva.

Clasificación JEL: D12, D14, M30, M31, L10, Z10.

\title{
Highlights
}

- El papel de la religiosidad conlleva a promover el consumo de productos que sean socialmente responsables.

- Basados en sus principios religiosos, y en su nivel de responsabilidad con el medio ambiente, son los consumidores quienes finalmente escogen la calidad del producto que van a consumir.

- La satisfacción momentánea y poco duradera del consumo de algunos bienes materiales nubla en los consumidores la decisión de realizar mejores compras.

\begin{abstract}
This study analyzes the influence of consumer religiosity, subjective norm, and perceived effectiveness on the intention to purchase socially responsible products. It applied empirical research based on a questionnaire administered to 485 participants in Colombia and Spain (divided in equal proportions). To evaluate the results, a structural equation model was created based on the Partial Least Squares (PLS) technique. The results of the two countries presented similarities; more specifically, religiosity influences the dimensions of responsible consumption in both places. In addition, the importance of the subjective norm in this type of consumption was confirmed. However, the hypothesis that related perceived consumer effectiveness and this kind of purchase was not supported. These results show the importance of the role of religiosity in responsible consumption intentions, mainly due to its symbolic and socializing function as an element of acculturation and expression of identity. Likewise, this paper expands the theory of planned behavior because it explores the subjective norm in this type of purchase. Further studies in this area should investigate responsible consumption and the factors that influence it and propose marketing strategies to strengthen it in favor of the environment, society, and the finances of organizations.
\end{abstract}

Keywords: Market structure, market behavior, perceived consumer effectiveness, subjective norm.

JEL classification: D12, D14, M30, M31, L10, Z10. 


\section{Highlights}

- As a result of the role religiosity plays in purchase intention, organizations should promote socially responsible products.

- Based on their religious principles and level of environmental responsibility, it is consumers who ultimately choose the quality of the product they will consume.

- The short-term satisfaction of consuming some material goods clouds consumers' decision to make better purchases.

\section{INTRODUCCIÓN}

El consumo socialmente responsable es un tema que hoy en día atrae a muchos investigadores para intentar explicar algunos rasgos en el consumidor (Prendergast \& Tsang, 2019; Roshani Perera y Rathnasiri Hewege, 2018; Jones et al., 2017) bajo la comprensión de que su comportamiento de compra no depende únicamente de las empresas, sino de como él asume su propia responsabilidad a la hora de adquirir productos (Bermeo-Giraldo et al., 2019; Baazeem et al., 2016). Entre muchas variables estudiadas, resulta interesante abordar la relación entre religiosidad y consumo responsable, la cual, en años recientes, ha recibido una atención creciente en la literatura académica (Millock et al., 2002; De Pelsmacker et al., 2005; Chai \& Tan, 2010; Felix \& Braunsberger, 2016; Sharma \& Sharma, 2017; Agarwala et al., 2019), debido principalmente a la coherencia con la visión ecológica del mundo que tiene la religiosidad y la necesidad de profundizar cada vez en su análisis.

Debido a esta necesidad, el presente trabajo busca explorar la relación entre la religiosidad, la norma subjetiva y la efectividad percibida del consumidor (EPC) y su influencia en el consumo responsable. También resulta importante abordar estos aspectos debido a la creciente preocupación por parte de las sociedades sobre los impactos ambientales y sociales que se derivan del modelo económico actual y de la insostenible gestión de los mismos en décadas recientes (Holotová et al., 2020; Davari et al., 2017; Khan \& Kirmani, 2018). Para François-Lecompte y Valette-Florence (2006) el consumo responsable se entiende como "el hecho de comprar productos que se percibe tiene un impacto positivo (o menos negativo) en el entorno y el uso del poder adquisitivo para expresar preocupaciones sociales y ambientales». Lo que implica, que tanto empresas como consumidores, empiezan a preocuparse por ofertar y demandar productos que sean social y ambientalmente responsables (Webb et al., 2008).

A la luz de lo expuesto, y para entender las relaciones planteadas, se analizaron 485 cuestionarios, aplicados en España y Colombia, teniendo en cuenta la identificación religiosa -en su mayoría católica-, con la intención de distinguir patrones comportamentales en los consumidores de estos países, aparentemente disímiles, que puedan influir en este tipo de consumo a favor del medio ambiente y de la sociedad (Van Raaij, 1978). La toma de muestras en dos países difiere de la mayoría de estudios en este campo, que utilizan muestras de un solo país (por ejemplo, Shamdasani et al., 1993; Teimourpour \& Heidarzadeh Hanzaee, 2011). Esto aumenta las probabilidades para que los resultados y sugerencias de investigación se consideren por parte de investigadores locales para profundizar en su desarrollo (Van Raaij, 1978). 
Influencia de la religiosidad, la norma subjetiva y la efectividad percibida del consumidor en el consumo socialmente responsable

Con esta investigación se pretende aportar una mayor comprensión sobre la influencia de la religiosidad en el consumo responsable. Además, se sugieren pautas a empresas para el manejo de productos de consumo responsable que promuevan en sus clientes decisiones que tengan un alto componente social y ambiental (Abutaleb et al., 2020). Y por último recomienda a las instituciones educativas y comunidades religiosas, interiorizar estos conceptos para realizar procesos de enseñanza-aprendizaje que permitan mejorar la calidad del consumo que hoy en día se produce (Abutaleb et al., 2020).

La estructura del presente documento contiene el marco conceptual con los interrogantes de la investigación, la descripción metodológica que se utilizó para conseguir los resultados, seguida de los hallazgos y la discusión para terminar con algunas conclusiones, limitaciones y líneas futuras de investigación.

\section{MARCO TEÓRICO}

\section{Consumo responsable}

El surgimiento de este concepto se presentó en la década de los sesenta para contrarrestar la visión del consumo normal, el cual puede ser entendido como la conciencia social de un individuo (Suárez et al., 2020) que tiene en cuenta las consecuencias públicas de su consumo privado como una construcción psicológica de participación social, donde, a través del poder adquisitivo, se pretende lograr un cambio en la sociedad, atendiendo su preocupación por los problemas sociales y ambientales (Lim et al., 2019), conocidos principalmente gracias a los movimientos medioambientalistas de la época (Webster, 1975).

Este tipo de consumo se puede resumir en tres dimensiones (Francois-Lecompte \& Roberts, 2006). La primera se denomina "Boicot a empresas irresponsables», la cual se refiere al grado en que un sujeto no compra a empresas por aspectos relacionados con sus comportamientos socialmente irresponsables. La segunda se ha designado con el nombre «Compra responsable y de proximidad», relacionada con el impacto de la compra con relación a causas sociales, apoyo a pequeñas empresas, origen geográfico y consumismo. Y la tercera se llama «Simplicidad voluntaria», que es el grado en que el individuo mide sus volúmenes de consumo, lo que necesita realmente y el grado de austeridad del gasto.

\section{Religiosidad}

El concepto de religiosidad lo han propuesto varios autores. En esta investigación se toma la de McDaniel y Burnett (1990), quienes afirman que es una creencia de que hay dios y hay principios rectores establecidos por él para los comportamientos y los acontecimientos de las personas en esta vida. A pesar de la multiplicidad de conceptos, debe ser revisada desde diferentes dimensiones como una fuerza cultural importante e influyente en la toma de decisiones del consumidor (Delener, 1990). De esta manera, mientras que la religión debe ser considerada como una variable demográfica, la religiosidad hace referencia a un rasgo de personalidad de los sujetos. Algunos estudios revelaron que la religión y la religiosidad tienen un efecto en las actitudes y comportamientos de los 
consumidores, ya que la religiosidad es un indicador de la religión de un individuo (Delener, 1990; Delener, 1994; McDaniel \& Burnett, 1990; Khraim, 2010).

\section{Efectividad percibida del consumidor}

La efectividad percibida del consumidor (EPC) hace referencia al juicio de una persona con respecto a la manera y el grado de impacto de su comportamiento de consumo respecto a la sociedad (Antil, 1984). Así, se relaciona con la creencia de que las acciones individuales contribuyen a la solución de los problemas sociales (Sharma \& Sharma, 2013; Straughan \& Roberts, 1999; Vermeir \& Verbeke, 2006), y funciona como una medida de la creencia del individuo con relación a sus acciones y como una estimación del grado en que las actividades de consumo personal contribuyen a la solución de un problema (Kinnear et al., 1974; Allen, 1982; Ellen et al., 1991). La EPC representa una evaluación de sí mismo en el contexto del problema (Berger \& Corbin, 1992) y resulta uno de los factores dominantes que influyen en las decisiones de compra, dependiendo del nivel de autoeficacia en el dominio especificado.

\section{Norma subjetiva}

La norma subjetiva

es un razonamiento normativo que pone de manifiesto la presión social percibida por la persona que va a realizar la conducta hacia la ejecución o no de dicha conducta; es el resultado de la evaluación que se hace, sobre si los otros significativos quieren que se realice la conducta y de la motivación para complacerlos. (Guzmán Facundo et al., 2014)

Este constructo se desprende en primera instancia de la teoría acción razonada y puede entenderse también como la percepción que tiene una persona de la presión social para actuar de cierta manera. Según esto, la norma subjetiva se ve determinada por la idea de aprobación y motivación para ajustarse a las expectativas de otros, lo que implica la influencia de características personales, comportamentales, de preferencia y demográficas (Ajzen \& Fishbein, 1980).

\section{Planteamiento de hipótesis}

\section{Religiosidad y consumo responsable}

Se podría esperar que los individuos altamente religiosos sean más dogmáticos y conservadores, alineándose de acuerdo con sus creencias y prácticas al apoyo de iniciativas empresariales de bajo impacto ambiental sugeridas por su afiliación religiosa (Chai \& Tan, 2010). Se sugiere, además, que aquellos que están fuertemente comprometidos con la religión son actitudinales, a la vez que conductuales, capaces de tomar decisiones consistentes con la conciencia moral, lo que resulta importante bajo la comprensión de que se evalúa la intención del individuo y esta no siempre es consistente con su forma de actuar (Sood \& Nasu, 1995).

Algunas investigaciones que apoyan dichas afirmaciones fueron las realizadas por Lau y Tan (2009) que revelaron que una persona con una alta religiosidad intrínseca sería más propensa a tener una actitud positiva hacia los productos ecológicos. Por su parte, Granzin y Olsen (1991) descubrieron 
Influencia de la religiosidad, la norma subjetiva y la efectividad percibida del consumidor en el consumo socialmente responsable

que las personas con un alto nivel de religiosidad tienden a reutilizar y reciclar los artículos usados. Rice (2006) descubrió el papel de la religiosidad en los comportamientos de compra ecológica en Egipto y los resultados mostraron que existe un vínculo positivo entre la religiosidad y los comportamientos ambientales, además de existir vínculos directos entre la religiosidad y los comportamientos de compra ecológica (Hassan, 2014; Mohamad et al., 2012). En contraste, Kalamas et al., (2014) no encontraron un vínculo entre la religiosidad y las compras verdes en Canadá, ni Kirmani y Khan (2016) encontraron un vínculo entre ambas variables en la India.

Estas investigaciones previas y el reconocimiento de que en su mayoría la religiosidad influye positivamente en el comportamiento de consumo responsable, permite proponer:

H1a:

H1a: En la Figura 1 se observa cómo la religiosidad tiene un efecto positivo en las dimensiones del consumo responsable: a) boicot a las empresas socialmente irresponsables, b) compra responsable y de proximidad, c) simplicidad voluntaria.

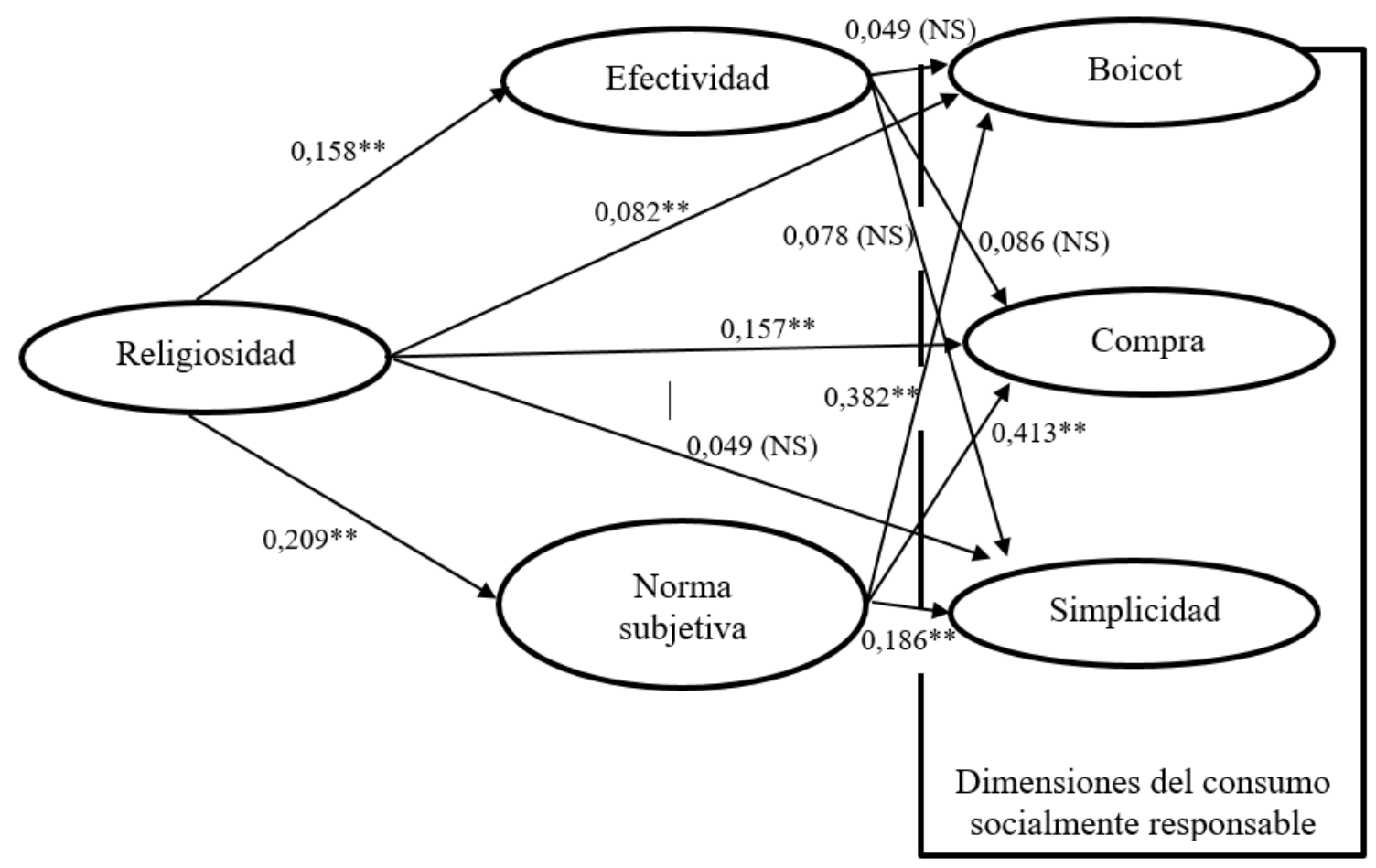

Figura 1. Resultados de las hipótesis

Figure 1. Model and hypothesis testing results Fuente: elaboración propia.

\section{Religiosidad, norma subjetiva y efectividad percibida del consumidor}

De acuerdo con la teoría de acción razonada (Ajzen \& Fishbein, 1980), los principales predictores de intenciones y comportamiento se derivan de creencias conductuales y normativas. Este enfoque, sin 
embargo, no aborda los orígenes de estas creencias, aunque si se acepta la definición de McDaniel y Burnett (1990) acerca de la religiosidad, en la medida en que las personas siguen los principios de su religión, habría más preocupación por las actitudes, comportamientos, normas y valores que por la afiliación en general (McAndrew \& Voas, 2011; Hoge, 1972). Por eso, la religiosidad puede influir en la norma subjetiva del individuo, debido a que las normas de comportamiento y los valores religiosos de una comunidad afectan la actitud y el comportamiento de las personas que hacen parte de la misma. Por ello, se propone:

H2: la religiosidad influye positivamente en la norma subjetiva (ver Figura 1).

Algunas investigaciones mencionan que si un sujeto percibe que sus acciones se ven afectadas por el grado en que cree que la ocurrencia o aversión de un evento puede verse afectada por estas, el sujeto, basado en sus creencias y valores (generalmente provistos por su religión), tenderá a actuar de manera positiva asumiendo que sus obras son beneficiosas para su entorno (Awad, 2011; Mas'od $\&$ Chin, 2014). En vista de estos esfuerzos que exploran los efectos combinados de la religiosidad, la sociodemografía y la psicografía, este estudio sugiere que la religiosidad es un determinante importante para explicar el comportamiento bajo la perspectiva de la EPC por el consumidor. Por lo tanto, se plantea la siguiente hipótesis:

H3: la religiosidad influye positivamente en la efectividad percibida del consumidor (ver Figura 1).

\section{Norma subjetiva y consumo responsable}

Bajo la teoría de acción razonada se podría pensar que la norma subjetiva que rodea el comportamiento de un individuo puede impactar su intención de actuar bajo el concepto de consumo responsable. Para Mohr et al., (2001) los consumidores responsables evitarán los productos o servicios de empresas que consideren perjudiciales para la sociedad y preferirán productos o servicios de empresas que la beneficien.

Dado el control suficiente sobre su comportamiento, se puede esperar que la gente intente al menos llevar a cabo sus intenciones, por lo tanto, la intención es el antecedente inmediato del comportamiento (Ajzen \& Fishbein, 1980). Después de haber creado una intención, el camino debe estar despejado para participar en el comportamiento real, es decir, si un consumidor tiene la intención de consumir de manera responsable, entonces debería ser muy probable que al menos trate de hacerlo (Prendergast \& Tsang, 2019). Contrariamente, Bray et al., (2011) argumentaron que las intenciones de los consumidores no siempre se traducen en un comportamiento de compra, tal vez porque la dimensión relacionada con una compra responsable se ve abrumada por otras cuestiones como el precio y la experiencia previa. De esta manera se decidió proponer el siguiente interrogante:

H4a: la norma subjetiva tiene un efecto positivo en las dimensiones del consumo responsable: a) boicot a las empresas socialmente irresponsables, b) compra responsable y de proximidad, c) simplicidad voluntaria. (ver Figura 1). 
Influencia de la religiosidad, la norma subjetiva y la efectividad percibida del consumidor en el consumo socialmente responsable

\section{Efectividad percibida del consumidor y consumo responsable}

La EPC se reconoce como un predictor útil del comportamiento de compra verde (Ellen, et al., 1991). De esa manera, algunos creen que la acción conduce a resultados particulares y, por lo tanto, produce cambios, mientras que otros tienen poca confianza en su capacidad para marcar la diferencia.

Aunque en un principio se consideraba como un elemento de la actitud y se modelaba como una variable moderadora (Kinnear et al.,1974; Webster, 1975; Antil, 1984), algunos investigadores propusieron que sería más efectivo hacerlo como construcciones distintas (Allen, 1982; Ellen et al., 1991). La revisión de literatura respalda la opinión de que la conciencia ambiental se manifiesta a través de las actitudes y creencias de los consumidores, tanto que sus acciones contribuyen a la solución de los problemas ambientales (Kinnear et al., 1974; Sharma \& Sharma, 2013; Straughan \& Roberts, 1999).

En línea con los argumentos anteriores, se selecciona esta variable para explorar la posible relación con la intención de compra responsable. Por lo tanto, se presume que:

H5a. La efectividad percibida del consumidor tiene un efecto positivo en las dimensiones del consumo responsable: a) boicot a las empresas socialmente irresponsables, b) compra de RSC de proximidad, c) simplicidad voluntaria (ver Figura 1).

\section{METODOLOGÍA}

\section{Muestra}

Se desarrolló un cuestionario único para recolectar datos empíricos que se envió aleatoriamente a través de diversas plataformas online, limitándose a Colombia y España para minimizar las diferencias religiosas dentro de la muestra. Según Orriols (2013), en España existe el dominio de la religión católica al igual que en Colombia (Bidegain Greising \& Demera Vargas, 2005), por lo que la distinción entre personas religiosas y no religiosas no es tan borrosa como en otros países o religiones (Halman et al., 2005). Una implicación de esta limitación geográfica es el hecho de que la mayoría de los encuestados son creyentes cristianos católicos practicantes y no practicantes.

El procedimiento de muestreo fue no probabilístico de conveniencia y bola de nieve. De los 730 cuestionarios distribuidos, 483 fueron completados y devueltos. De este tamaño muestral, el 50.9\% son colombianos, la edad promedio de la muestra fue de 39 años (DE $=12.5$ años), el $51.6 \%$ fueron mujeres. Con relación al nivel académico, el $37.1 \%$ son universitarios, el $34.1 \%$ cuentan con posgrado, el $27.1 \%$ termino la secundaría y los faltantes únicamente terminaron primaria. De los participantes, $71.5 \%$ se definieron cristianos católicos, el $22.2 \%$ no son religiosos y el $5 \%$ indicó estar afiliado al cristianismo protestante. De estos, el $44.6 \%$ se considera practicante y el $35 \%$ no practicante de los rituales propios de su religión. 


\section{Escalas empleadas}

Todas las escalas se evaluaron con una escala tipo Likert de 7 puntos, de (1) totalmente en desacuerdo a (7) totalmente de acuerdo y se presentan en el apéndice 1. Para medir la religiosidad se evaluaron diferentes artículos relacionando esta variable con el comportamiento del consumidor responsable, se eligió la escala propuesta por Mohd Dali et al., (2019) debido a que contempla aspectos que tienen que ver con las creencias, compromiso, práctica y la influencia de la religiosidad en las personas, algunos de ellos relacionadas con el consumo. Sin embargo, se eliminó aquellos ítems que no correspondían a la religión predominante en los países en estudio. El encabezado de la pregunta fue: «en relación con su creencia principal...» y luego se sugerían las afirmaciones para ser evaluadas. Tanto fiabilidad compuesta como varianza extraída resultaron ser buenas para esta escala $(\mathrm{CR}=0.939 \mathrm{AVE}=0.537)($ ver Tabla 1$)$.

Para medir la efectividad percibida del consumidor se adaptó para este estudio la escala de Straughan y Roberts (1999) debido a la alta correlación verificada en sus estudios. El encabezado del apartado consistió en formular el siguiente cuestionamiento: "cuando realizo mis compras...», seguido de los ítems de evaluación. Para esta escala se obtuvo unos buenos índices de fiabilidad compuesta y varianza extraída $(\mathrm{CR}=0.745 \mathrm{AVE}=0.640)$ (ver Tabla 1$)$.

La norma subjetiva se midió con la escala original de Ajzen y Fishbein (1980) adaptada para este estudio, puesto que dicha norma está planteada en la teoría de estos autores como una variable que determina la intención de uso o compra. Para presentar el cuestionario se utilizó como encabezado: "cuando realizo mis compras...», seguido de los ítems de evaluación. Los resultados de fiabilidad compuesta y varianza extraída fueron aceptables $(C R=0.797$ AVE $=0.505)$ (ver Tabla 1$)$.

Por último, se tomó la escala de François-Lecompte y Valette-Florence (2006) para medir el consumo responsable, la razón fue que los autores analizaron la variable multidimensionalmente, lo que permitió dividir en tres la intención de consumo responsable para efectos de modelización. De esta manera se toman tres de las cinco dimensiones que proponen los autores, las cuales se renombraron como: boicot a las empresas socialmente irresponsables (BESI), compra responsable y de proximidad (CRP) y simplicidad voluntaria (SV), lo que permitió un estudio predictivo detallado de la intención de compra. Para evaluar esta dimensión se cuestionó: «usted ha intentado», seguido a esto se ubicaron las afirmaciones correspondientes. Los resultados de fiabilidad compuesta y varianza extraída fueron aceptables para las tres dimensiones $\left(\mathrm{CR}_{\mathrm{BESI}}=0.864 \mathrm{AVE}_{\mathrm{BESI}}=0.679 ; \mathrm{CR}_{\mathrm{CRP}}=0.845 \mathrm{AVE}_{\mathrm{CRP}}=0.525 \mathrm{CR}_{\mathrm{SV}}\right.$ $\left.=0.865 \mathrm{AVE}_{\mathrm{sv}}=0.763\right)$ (ver Tabla 1).

Para abordar las posibles preocupaciones del sesgo de método común y el sesgo de fuente común se recurrió a los remedios propuestos por Podsakoff et al., (2003), para lo cual una misma persona no podía responder el instrumento más de una vez y se hizo necesario proteger la privacidad de los encuestados, asegurándoles que no había respuestas correctas o incorrectas y que debían responder de la manera más honesta posible recordándoles que los datos recabados se utilizarían solo con fines de investigación.

Antes de aplicar el cuestionario definitivo se realizó una prueba piloto que confirmara que los ítems no se redactaron de manera incomprensible o con términos vagos, ambiguos o que pudieran no ser comprendidos, eliminando el sesgo de aquiescencia (Winkler et al., 1982). Para esto se tradujeron las 
Influencia de la religiosidad, la norma subjetiva y la efectividad percibida del consumidor en el consumo socialmente responsable

preguntas del idioma original (francés e inglés), manteniendo el ítem simple, pero sin perder la esencia. Además, se separaron los elementos de la escala con el afán de reducir la probabilidad de que los encuestados adivinen la relación entre las variables dependientes e independientes y que conscientemente correspondan sus respuestas a las diferentes medidas (Fink, 2003), por loa cual se apostó a realizar una encuesta online aleatoria. Una razón final para creer que hay un posible sesgo de aquiescencia es que se encontró una gran variación en los puntajes de los diversos componentes de la EPC, sin embargo, esto no es predominante.

Para sustentar la decisión sobre el algoritmo de estimación del modelo a utilizar, se evaluó tanto el objetivo, los indicadores, constructos y el modelo definido, para finalmente optar por aplicar la técnica multivariante de ecuaciones estructurales con enfoque en PLS. Este algoritmo se orienta al estudio predictivo en situaciones de alta complejidad y con escaso conocimiento teórico, un tamaño de muestra no muy grande teniendo en cuenta que se hizo en dos países, y que luego de varias pruebas no se pudo determinar una normalidad en la distribución de los datos (Wold, 1975; Hair et al., 1998; Chin et al., 1998).

\section{RESULTADOS}

Para contrastar las hipótesis del modelo, la Figura 1 muestra las relaciones esperadas bajo las cuales el software que se utilizó estima simultáneamente los parámetros de medida estructurales. Los resultados se interpretaron en dos sentidos: primero, evaluando las escalas de medidas, y segundo, evaluando el modelo estructural (Anderson \& Gerbing, 1988). El software utilizado para la estimación de los parámetros fue el Smart-PLS 3.0 (Ringle et al., 2018), utilizando un bootstrapping de 500 muestras para el cálculo de la significatividad de los parámetros.

En este modelo, la religiosidad actúa como variable independiente, mientras que las otras variables son dependientes. En primera instancia, se determinó el índice de fiabilidad compuesta (CR), que permite medir la consistencia interna del bloque de indicadores, es decir de los indicadores que analizan las variables latentes (Lévy Mangin \& Varela, 2006), más adecuada que el alfa de Cronbach para PLS al no asumir que todos los indicadores reciben la misma ponderación (Chin, 1998), por lo cual no se incluye el alfa de Cronbach en las tablas posteriores. Los autores sugieren validar con un valor de al menos 0.7, considerado como un nivel modesto principalmente para investigaciones exploratorias (Fornell \& Larcker 1981; Nunnally \& Bernstein, 1994), cuestión que queda zanjada para todos los constructos evaluados (ver Tabla 1 ).

Seguido de este aparece la validez convergente, para lo cual se calculó el índice de varianza extraída (AVE) de cada constructo, respectivamente, y cuyo valor sugerido debe ser mayor a 0.5 (Fornell \& Larcker, 1981) que, como se ve en la Tabla 1, es superado por todas las variables. El AVE que mide «la cantidad total de la varianza de los indicadores tenida en cuenta por el constructo latente» (Lévy Mangin \& Varela, 2006, p. 130). 
Tabla 1. Consistencia interna y validez convergente del instrumento de medida

Table 1. Internal consistency and convergent validity of the measurement instrument

\begin{tabular}{|c|c|c|c|c|c|}
\hline Factor & Indicador & L & $\mathrm{t}$ & CR & AVE \\
\hline \multirow{11}{*}{ Religiosidad } & $\begin{array}{l}\text {... los valores espirituales son más importantes } \\
\text { que las cosas materiales. }\end{array}$ & 0.634 & 15.17 & 0.939 & 0.587 \\
\hline & ... mi relación con Dios es importante para mí. & 0.781 & 29.47 & & \\
\hline & ... busco mi fe como una fuente de inspiración. & 0.863 & 50.17 & & \\
\hline & $\begin{array}{l}\text {... las celebraciones religiosas son importantes } \\
\text { para mí. }\end{array}$ & 0.795 & 28.29 & & \\
\hline & $\begin{array}{l}\text {... tengo el conocimiento básico y necesario } \\
\text { sobre mi religión. }\end{array}$ & 0.679 & 20.51 & & \\
\hline & $\begin{array}{l}\text {... es importante para mí pasar el tiempo en } \\
\text { pensamientos privados y oración. }\end{array}$ & 0.828 & 47.31 & & \\
\hline & $\begin{array}{l}\text {... me esfuerzo por vivir toda mi vida de } \\
\text { acuerdo a mis creencias religiosas. }\end{array}$ & 0.851 & 37.16 & & \\
\hline & $\begin{array}{l}\text {... muchos aspectos de mi vida están basados } \\
\text { en mi religión. }\end{array}$ & 0.902 & 69.38 & & \\
\hline & $\begin{array}{l}\text {... mis creencias religiosas influyen en lo que } \\
\text { compro. }\end{array}$ & 0.679 & 16.36 & & \\
\hline & $\begin{array}{l}\text {... mis creencias religiosas influyen sobre qué } \\
\text { proveedores de servicio uso, confiando en sus } \\
\text { valores religiosos. }\end{array}$ & 0.602 & 12.09 & & \\
\hline & ... voy a lugares de culto regularmente & 0.750 & 24.35 & & \\
\hline \multirow[b]{2}{*}{ Efectividad } & $\begin{array}{l}\text {... el modo en que me comporto como } \\
\text { consumidor afecta el medio ambiente. }\end{array}$ & 0.641 & 7.80 & 0.775 & 0.640 \\
\hline & $\begin{array}{l}. . \text { cada consumidor puede tener una } \\
\text { influencia positiva en la sociedad a través de la } \\
\text { compra de productos de empresas } \\
\text { socialmente responsables. }\end{array}$ & 0.933 & 32.85 & & \\
\hline \multirow{4}{*}{ Norma } & $\begin{array}{l}\text {... la mayoría de mis amigos esperan que yo } \\
\text { proteja el medio ambiente. }\end{array}$ & 0.692 & 17.79 & 0.797 & 0.505 \\
\hline & $\begin{array}{l}\text {... la mayoría de las personas que son } \\
\text { importantes para mí piensan que yo debería } \\
\text { comprar productos de consumo socialmente } \\
\text { responsables cuando voy de compras. }\end{array}$ & 0.785 & 29.40 & & \\
\hline & $\begin{array}{l}\text {... la mayoría de las personas que son } \\
\text { importantes para mí deberían comprar } \\
\text { productos de consumo socialmente } \\
\text { responsable cuando van de compras. }\end{array}$ & 0.836 & 40.19 & & \\
\hline & $\begin{array}{l}\text {... la opinión positiva de mis amigos influye en } \\
\text { mi compra de productos de consumo } \\
\text { socialmente responsables. }\end{array}$ & 0.478 & 7.77 & & \\
\hline \multirow{3}{*}{ Boicot } & $\begin{array}{l}\text {... no comprar productos fabricados por } \\
\text { empresas que utilizan trabajo infantil. }\end{array}$ & 0.767 & 24.58 & 0.864 & 0.679 \\
\hline & $\begin{array}{l}. . \text { no comprar a compañías que tienen } \\
\text { prácticas irrespetuosas hacia sus empleados. }\end{array}$ & 0.860 & 42.16 & & \\
\hline & ... no comprar a compañías que contaminan. & 0.843 & 43.45 & & \\
\hline Compra & $\begin{array}{l}\text {... comprar productos cuya producción sea } \\
\text { sostenible en favor del medio ambiente. }\end{array}$ & 0.826 & 50.06 & 0.845 & 0.525 \\
\hline
\end{tabular}


Influencia de la religiosidad, la norma subjetiva y la efectividad percibida del consumidor en el consumo socialmente responsable

\begin{tabular}{|c|c|c|c|c|c|}
\hline & $\begin{array}{l}\text {... comprar productos por los cuales parte del } \\
\text { precio se dona a una buena causa. }\end{array}$ & 0.649 & 16.77 & & \\
\hline & $\begin{array}{l}\text {... apoyar a los comerciantes de mi vecindario } \\
\text { a través de mis compras. }\end{array}$ & 0.763 & 25.43 & & \\
\hline & $\begin{array}{l}. . \text { ir al mercado para apoyar a los pequeños } \\
\text { productores de frutas y verduras. }\end{array}$ & 0.673 & 16.91 & & \\
\hline & ... comprar productos hechos en mi región. & 0.699 & 17.52 & & \\
\hline \multirow{2}{*}{ Simplicidad } & $\begin{array}{l}\text {... limitar mi consumo a lo que realmente } \\
\text { necesito. }\end{array}$ & 0.925 & 35.04 & 0.865 & 0.763 \\
\hline & ... en general, no consumir demasiado. & 0.819 & 15.34 & & \\
\hline
\end{tabular}

En la Tabla 2 se muestra el índice heterotrait-monotrait (HTMT), que permite confirmar la validez discriminante que estuvo por debajo de 0.90, ya que el promedio de las correlaciones entre indicadores que miden constructos distintos fueron menores del punto de corte establecido en todos los constructos como sugieren los autores (Gold et al., 2001).

Tabla 2. Validez discriminante

Table 2. Discriminant validity

\begin{tabular}{ccccccc}
\hline Factor & F1 & F2 & F3 & F4 & F5 & F6 \\
\hline F1 Religiosidad & 0.766 & 0.175 & 0.236 & 0.177 & 0.286 & 0.123 \\
\hline F2 Efectividad & 0.159 & 0.800 & 0.772 & 0.392 & 0.483 & 0.274 \\
\hline F3 Norma & 0.209 & 0.519 & 0.711 & 0.469 & 0.568 & 0.311 \\
\hline F4 Boicot & 0.170 & 0.261 & 0.425 & 0.824 & 0.777 & 0.328 \\
\hline F5 Compra & 0.257 & 0.325 & 0.491 & 0.619 & 0.725 & 0.476 \\
\hline F6 Simplicidad & 0.101 & 0.183 & 0.238 & 0.250 & 0.349 & 0.873 \\
\hline Nota: diagonal, raíz cuadrada de la varianza extraída. Triángulo inferior: correlaciones entre las variables latentes. \\
& $\begin{array}{c}\text { Triángulo superior: ratio HTMT } \\
\text { Fuente: elaboración propia. }\end{array}$ & & \\
& \multicolumn{7}{c}{} & &
\end{tabular}

La Tabla 3 recoge los valores de los coeficientes estandarizados de las relaciones estructurales y los respectivos niveles de significatividad de su estadístico $t$ asociado (Hair et al., 1998). Cabe destacar que de las 11 hipótesis planteadas se descartan todas las relacionadas con la EPC y la relación entre la religiosidad y la simplicidad voluntaria. Tal como se observa en la Figura 1, las relaciones más fuertes se presentan entre la norma subjetiva y las dimensiones del consumo responsable, seguido por la religiosidad, además se puede denotar los valores bajos y la imposibilidad de comprobar las hipótesis relacionadas con la EPC. 
Tabla 3. Estimación del modelo estructural

Table 3. Estimation of the structural model

\begin{tabular}{ccc}
\hline Hipótesis & B estandarizado & Valor $t$ \\
\hline H1a. Religiosidad --> Boicot & $0.082^{* *}$ & 2.00 \\
\hline H1b. Religiosidad --> Compra & $0.157^{* *}$ & 3.68 \\
\hline H1c. Religiosidad --> Simplicidad & 0.049 & 1.03 \\
\hline H2. Religiosidad --> Efectividad & $0.158^{* *}$ & 3.78 \\
\hline H3. Religiosidad --> Norma & $0.209^{* *}$ & 4.81 \\
\hline H4a. Efectividad --> Boicot & 0.049 & 0.97 \\
\hline H4b. Efectividad --> Compra & 0.086 & 1.82 \\
\hline H4c. Efectividad --> Simplicidad & 0.078 & 1.49 \\
\hline H5a. Norma --> Boicot & $0.382^{* *}$ & 7.15 \\
\hline H5b. Norma --> Compra & $0.413^{* *}$ & 9.66 \\
\hline H5c. Norma --> Simplicidad & $0.186 * *$ & 3.53 \\
\hline R2 (efectividad) = 0.0253; R2 (norma) $=0.0437 ;$ R2 (organización) $=0.2713 ;$ R2 (compra) $=0.2713 ;$ R2 (volumen) $=0.0638 ;$ \\
Q2 (efectividad)=0.115; Q2 (norma) = 0.019; Q2 (organización) $=0.118 ;$ Q2(volumen) $=0.040 ;{ }^{* * p}<0.01 ;{ }^{*}<<0.05$ \\
Fuente: elaboración propia.
\end{tabular}

\section{DISCUSIÓN}

En primer lugar, el efecto de la religiosidad sobre boicot a empresas socialmente irresponsables y la compra responsable y de proximidad resultó ser significativo, no pudiéndose rechazar la H1a y b. Este hallazgo está en línea con trabajos previos, donde se sugiere la probabilidad de que un consumidor religioso apoye las iniciativas de responsabilidad social de las compañías, como comprar a aquellas que sean respetuosas con sus empleados y que no contraten niños en el trabajo (Angelidis, \& Ibrahim, 2004; Chai \& Tan, 2010; Akremi \& Smaoui, 2015). También es importante que el consumidor intente minimizar en su compra el impacto medio ambiental, comprando a vendedores pequeños, locales y cercanos, atendiendo a que los seguidores religiosos atribuyen a ellos mismos y a los productores la responsabilidad del bienestar medioambiental (Leary et al., 2016; Mohd Dali et al., 2019). Además, tener en cuenta que esta preocupación por el medio ambiente se rige por valores centrados en el ser humano a partir de sus creencias, asumiendo la omnipresencia de la naturaleza (Mayer \& Frantz, 2004).

Luego, según los resultados, se debe rechazar la H1c, ya que la religiosidad no influye en la simplicidad voluntaria; este hallazgo, aunque relativamente contradictorio, podría atribuirse a dos razones: por un lado, teniendo en cuenta como la religión afirma la identidad de un individuo y la enorme necesidad por conseguir el reconocimiento social por parte de este, lo que no le permite ser conservador en sus compras (Aguirre, 1988; Woodhead, 2011); por el otro, en la forma en que se fomenta, facilita y estimula el reciclaje en los países evaluados que implicaría que la cantidad consumida «no impacta» el medio ambiente (Anderson et al., 1974).

En segundo lugar, la religiosidad influye en la EPC (aceptación de H2). Con relación a esta última, un consumidor se puede ver influenciado por su nivel de religiosidad en cuanto lo relevante que sean 
Influencia de la religiosidad, la norma subjetiva y la efectividad percibida del consumidor en el consumo socialmente responsable

sus valores y creencias para influir con su comportamiento en las actuaciones de la sociedad y en su actitud para solucionar un problema (Hunt \& Vitell, 2006).

En tercer lugar, la religiosidad influye en la norma subjetiva (aceptación de H3), esto se muestra acorde con la literatura, donde se afirma que los valores y las normas implican la práctica de la austeridad en el consumo (Aguirre, 1988). Además, muestra que los miembros de una comunidad religiosa con altos niveles de participación en actividades formales tienden a ser más interdependientes y dogmáticos (Rokeach, 1969), esto permite argumentar que existe una presión social por parte de la comunidad religiosa que puede influir en el comportamiento de un individuo. Asimismo, Cosgel y Minkler (2004) sugieren que las normas de una religión facilitan la elección de ciertos productos $y$, al ser un marco grupal, intensifican y ayudan a expresar mejor esas creencias.

En cuarto lugar, los resultados del presente estudio confirman la influencia de la norma subjetiva en la intención de consumo responsable (aceptación de $\mathrm{H} 4 \mathrm{a}, \mathrm{b}$ y c). Este hallazgo aporta a la teoría del comportamiento planificado, que junto con la actitud son predictores de la intención de comportamiento (Ajzen \& Fishbein, 1980; Ajzen, 1991); además, la presión social ejercida sobre el consumidor puede ser de particular interés en el comportamiento ambiental, porque tanto familia, socios, comunidad (religiosa), niños, así como el mismo sujeto, pueden ejercerla en favor de un consumo responsable (Ekström, 1995; Bratt, 1999).

Por último, en relación con las hipótesis rechazadas sobre la influencia de la EPC sobre la intención de consumo responsable (rechazo de $\mathrm{H} 5 \mathrm{a}, \mathrm{b}$ y $\mathrm{c}$ ), se está en consonancia con el hecho de que los consumidores no se sienten capaces de traducir sus preocupaciones generales a acciones privadas por el bajo nivel de autoeficacia percibida con relación al consumo; esto demuestra que el individuo puede asumir un alto grado de impotencia percibida que podría verse reflejada en los resultados obtenidos, dando a entender que el sujeto no se siente capaz de gestar cambios en el medio ambiente a partir de su consumo (Berger \& Corbin, 1992).

\section{CONCLUSIONES}

El actual documento pretende contribuir a la literatura al destacar el papel de la religiosidad en las intenciones de consumo responsable, en su función simbólica y socializadora como elemento de aculturación y de expresión de identidad, que sugiere a los individuos, no solo una felicidad basada en lo material, sino en lo espiritual (Vela \& Ballesteros, 2011). Los autores consideran la religiosidad como una parte clave para la consecución de valores y creencias, por lo tanto, tiene una influencia significativa en los individuos, tanto como seres humanos, como consumidores. Por ello, la religión como tal, siempre tiene algo que decir sobre el consumo (Katz-Gerro \& Meier Jaeger, 2012).

Además, sobre la religiosidad y la religión, es importante concluir también que su influencia en el consumo responsable puede ser inspiradora, aunque también una fuente de tensión (Mazereeuw der Duijn Schouten et al., 2014). Por un lado, puede inspirar a poner en práctica valores religiosos que estén relacionados con este tipo de consumo en las compras cotidianas. Sin embargo, puede, en algún punto, reafirmar la difícil situación que hoy en día tienen las iglesias para convencer a sus seguidores de que obren de determinada manera, lo que resulta en dilemas y conflictos para ambas partes. 
Si bien algunas religiones han sido más intensas y dogmáticas a la hora de introducir sugerencias en el consumo (Mandeville, 2001), en las costumbres cristianas no hay restricciones específicas, lo que permite un enfoque más liberal al individuo en ese sentido (Murphy, 2014). Esto deja en él la posibilidad de decidir, con base en sus creencias y muchos otros factores, la compra que realiza. Por ende, no se podría sugerir que sea la religión la que no realiza sus esfuerzos por mejorar los hábitos de compra en sus feligreses, sino son ellos quienes los determinan, basados en estos y otros principios que pueden mejorar o empeorar el tipo de consumo.

Esta investigación también amplia la teoría del comportamiento planificado, especialmente al tomar la norma subjetiva como antecedente de la intención de consumo (Ajzen, 1991). De hecho, los resultados relacionan fuertemente esta variable con las tres dimensiones planteadas en este estudio, bajo el criterio que, para el cristiano la realidad material es buena y está al servicio de él y no se debería demonizar, sino discernirse desde la norma personal y moral de la creencia y la libertad otorgada por la misma (Aguirre, 1988).

Teniendo en cuenta los pobres resultados que arrojó la efectividad percibida del consumidor sobre la intención de consumir responsablemente, se puede concluir que las entidades encargadas por parte del Estado deberían promover comportamientos individuales que convenzan a los ciudadanos de su propia autoeficacia, y como ellos, además de preocuparse por el impacto medio ambiental y social, pueden ser personalmente agentes de cambio para combatir los problemas ambientales. Los consumidores necesitan poder para confiar en sus propias capacidades y así lograr resultados ambientales valiosos (Berger \& Corbin, 1992).

Asimismo, la «falacia del consumismo», como la gran religión popular de las sociedades desarrolladas, seduce irremediablemente a las personas para realizar compras sin sentido y para ello utiliza varios mecanismos y excusas para lograrlo (status, reconocimiento, publicidad, entre otras) (Aguirre, 1988). Bajo esta concepción, esta investigación no se permite proponer un cambio en el consumo, pero si un repensar de él, a partir de la idea de que la satisfacción momentánea y poco duradera de los bienes materiales nubla las decisiones. Sin embargo, cada individuo, al evaluar de manera responsable sus compras, puede lograr un cambio que favorezca, no solo su consumo y su vida, sino también la vida de los demás.

Por último, esta investigación invita a personas interesadas por temas que combinen la religión con el estudio de los mercados, a considerar la evaluación de otras variables para entender el consumo responsable, determinando que el valor del presente artículo se encuentra detrás de considerar únicamente tres variables. Igualmente es importante, como bien se pudo notar en el estudio, proponer que la variable EPC sea analizada como moderadora, pudiendo, en opinión de los autores, extraer mejores resultados.

\section{CONFLICTOS DE INTERÉS}

Los autores declaran que no presentan conflictos de interés financiero, profesional o personal que pueda influir de forma inapropiada en los resultados obtenidos o las interpretaciones propuestas. 
Influencia de la religiosidad, la norma subjetiva y la efectividad percibida del consumidor en el consumo socialmente responsable

\section{CONTRIBUCIÓN DE AUTORES}

Para el desarrollo de este proyecto todos los autores han realizado una contribución significativa especificada a continuación:

Juan Camilo Mejía: conceptualización, diseño y desarrollo de la investigación, redacción y revisión final del manuscrito.

Rafael Currás-Pérez: conceptualización, diseño y desarrollo de la investigación y revisión final del manuscrito.

Carlos Manuel Córdoba-Segovia: conceptualización, diseño y desarrollo de la investigación y revisión final del manuscrito.

\section{REFERENCIAS}

Abutaleb, S.; El-Bassiouny, N. M.; Hamed, S. (2020). A conceptualization of the role of religiosity in online collaborative consumption behavior. Journal of Islamic Marketing, v. 12, n. 1, 180-198. https://doi.org/10.1108/JIMA-09-2019-0186

Agarwala, R.; Mishra, P.; Singh, R. (2019). Religiosity and consumer behavior: A summarizing review. Journal of Management, Spirituality and Religion, v. 16, n. 1, 32-54. https://doi.org/10.1080/14766086.2018.1495098

Aguirre, R. (1988). El evangelio como juicio a la cultura del consumo. Sal Terrae, v. 4, 265-274.

Ajzen, H.; Fishbein, M. (1980). Predicting and Understanding Consumer Behavior: Attitude-Behavior Correspondence. En Ajzen, I.; Fishbein, M. (Eds.). Understanding Attitudes and Predicting Social Behavior (148-172). Prentice Hall.

Ajzen, I. (1991). The theory of planned behavior. Organizational behavior and human decision processes, v. 50, n. 2, 179-211. https://doi.org/10.1016/0749-5978(91)90020-T

Akremi, A.; Smaoui, F. (2015). Socially responsible consumption in emerging markets: ¿do cultural values and religiosity matter? En Vrontis, D.; Weber, Y; Tsoukatos, E. (Eds.), 8th Annual Conference of the EuroMed Academy of Business (p. 46-83). EuroMed Press.

Allen, C. T. (1982). Self-Perception Based Strategies for Stimulating Energy Conservation. Journal of Consumer Research, v. 8, n. 4, 381-390. https://doi.org/10.1086/208878

Anderson, J.; Gerbing, D. (1988). Structural equation modeling in practice: A review and recommended two-step approach. Psychological bulletin, v. 103, n. 3, 411. URL 
Anderson, W. T.; Henion, K. E.; Cox, E. P. (1974). Socially vs. ecologically responsible consumers. En AMA Combined Conference Proceedings (v. 36, pp. 304-311).

Angelidis, J.; Ibrahim, N. (2004). An exploratory study of the impact of degree of religiousness upon an individual's corporate social responsiveness orientation. Journal of business ethics, n. 51, 119128. https://doi.org/10.1023/B:BUSI.0000033606.27489.bf

Antil, J. H. (1984), Socially Responsible Consumers: Profile and Implications for Public Policy. Journal of Macromarketing, v. 4, n. 2, 18-39. https://doi.org/10.1177/027614678400400203

Awad, T. A. (2011). Environmental segmentation alternatives: buyers' profiles and implications. Journal of Islamic Marketing, v. 2, n. 1, 55-73. https://doi.org/10.1108/17590831111115240

Baazeem, T.; Mortimer, G.; Neale, L. (2016). Conceptualising the relationship between shopper religiosity, perceived risk and the role of moral potency. Journal of Consumer Behaviour, v. 15, n. 5, 440-448. https://doi.org/10.1002/cb.1583

Berger, I. E.; Corbin, R. M. (1992). Perceived consumer effectiveness and faith in others as moderators of environmentally responsible behaviors. Journal of Public Policy \& Marketing, v. 11, n. 2, 7989. https://doi.org/10.1177/074391569201100208

Bermeo-Giraldo, M. C.; Álvarez-Agudelo, L.; Ospina-Rúa, M. I.; Acevedo-Correa, Y.; MontoyaRestrepo, I. A. (2019). Factores que influyen en la intención de uso de las tarjetas de crédito por parte de los jóvenes universitarios. Revista CEA, v. 5, n. 9, 77-96.

https://doi.org/10.22430/24223182.1257

Bidegain Greising, A. M.; Demera Vargas, J. D. (2005). Globalización y diversidad religiosa en Colombia (Vol. 34). Universidad Nacional de Colombia.

Bratt, C. (1999). The impact of norms and assumed consequences on recycling behavior. Environment and behavior, v. 31, n. 5, 630-656. https://doi.org/10.1177/00139169921972272

Bray, J.; Johns, N.; Kilburn, D. (2011). An exploratory study into the factors impeding ethical consumption. Journal of Business Ethics, v. 98, n. 4, 597-608.

https://doi.org/10.1007/s10551-010-0640-9

Chai, L. T.; Tan, B. C. (2010). Towards socially responsible consumption: Investigating the effect of religiosity and money ethics. International Journal of Trade, Economics and Finance, v. 1, n. 1, 32-35. https://doi.org/10.7763/IJTEF.2010.V1.6

Chin, W. W. (1998). The partial least squares approach to structural equation modeling. En Marcoulides, G. A. (Ed.), Modern methods for business research (295-358). Lawrence Erlbaum.

Cosgel, M. M.; Minkler, L. (2004). Religious identity and consumption. Review of Social Economy, v. 62, n. 3, 339-350. https://doi.org/10.1080/0034676042000253945 
Influencia de la religiosidad, la norma subjetiva y la efectividad percibida del consumidor en el consumo socialmente responsable

Davari, A.; Iyer, P.; Strutton, D. (2017). Investigating moral links between religiosity, altruism, and green consumption. Journal of Nonprofit \& Public Sector Marketing, v. 29, n. 4, 385-414. https://doi.org/10.1080/10495142.2017.1326338

De Pelsmacker, P.; Driesen, L.; Rayp, G. (2005). Do consumers care about ethics? Willingness to pay for fair-trade coffee. Journal of consumer affairs, v. 39, n. 2, 363-385. https://doi.org/10.1111/j.1745-6606.2005.00019.x

Delener, N. (1990). The Effects of Religious Factors on Perceived Risk in Durable Goods Purchase Decisions. Journal of Consumer Marketing, v. 7, n. 3, 27-38. https://doi.org/10.1108/EUM0000000002580

Delener, N. (1994). Religious contrasts in consumer decision behaviour patterns: their dimensions and marketing implications. European Journal of Marketing, v. 28, n. 5, 36-53. https://doi.org/10.1108/03090569410062023

Ekström, K. M. (1995). Children's influence in family decision making: A study of yielding, consumer learning, and consumer socialization. [Tesis de doctorado, Göteborg University]. URL

Ellen, P. S.; Wiener, J. L.; Cobb-Walgren, C. (1991). The role of perceived consumer effectiveness in motivating environmentally conscious behaviors. Journal of public policy \& marketing, v. 10, n. 2, 102-117. https://doi.org/10.1177/074391569101000206

Felix, R.; Braunsberger, K. (2016). I believe therefore I care: the relationship between religiosity, environmental attitudes, and green product purchase in Mexico. International Marketing Review, v. 33, n. 1, 137-155. https://doi.org/10.1108/IMR-07-2014-0216

Fink, A. (2003). The survey handbook. Sage Publications.

Fornell, C; Larcker, D. F. (1981). Evaluating structural equation models with unobservable variables and measurement error. Journal of Marketing Research, v. 18, n. 1, 39-50. https://doi.org/10.1177/002224378101800104

François-Lecompte, A.; Valette-Florence, P. (2006). Mieux connaître le consommateur socialement responsable. Décisions Marketing, n. 41, 67-80. $\underline{\text { URL }}$

Gold, A.; Malhotra, A.; Segars, A. H. (2001). Knowledge management: an organizational capabilities perspective. Journal of Management Information Systems, v. 18, n. 1, 185-214. https://doi.org/10.1080/07421222.2001.11045669

Granzin, K. L.; Olsen, J. E. (1991). Characterizing participants in activities protecting the environment: A focus on donating, recycling, and conservation behaviors. Journal of Public Policy \& Marketing, v. 10, n. 2, 1-27. https://doi.org/10.1177/074391569101000201 
Guzmán Facundo, F. R.; García Salas, B. A.; Rodríguez Aguilar, L.; Alonso Castillo, M. M. (2014). Actitud, norma subjetiva y control conductual como predictores del consumo de drogas en jóvenes de zona marginal del norte de México. Frontera Norte, v. 26, n. 51, 53-74. https://doi.org/10.17428/rfn.v26i51.556

Hair, J. F.; Anderson, R. E.; Tatham, R. L.; Black, W. C. (1998). Multivariate data analysis. Englewood Cliffs, NJ.

Halman, L.; Luijkx, R.; Van Zundert, M. (2005). Atlas of European Values (European values studies). Brill Academic Pub.

Hassan, S.H. (2014). The role of Islamic values on green purchase intention. Journal of Islamic Marketing, v. 5, n. 3, 379-395. https://doi.org/10.1108/JIMA-11-2013-0080

Hoge, R. (1972). A validated intrinsic religious motivation scale. Journal for the Scientific Study of Religion, v. 11, n. 4, 369-376. https://doi.org/10.2307/1384677

Holotová, M.; Nagyová, Ĺ.; Holota, T. (2020). The impact of environmental responsibility on changing consumer behaviour-sustainable market in Slovakia. Economics \& Sociology, v. 13, n. 3, 84-96. https://doi.org/10.14254/2071-789X.2020/13-3/6

Hunt, S. D.; Vitell, S. J. (2006). The general theory of marketing ethics: A revision and three questions. Journal of macromarketing, v. 26, n. 2, 143-153. https://doi.org/10.1177/0276146706290923

Jones, J. J.; Reilly, T. M.; Cox, M. Z.; Cole, B. M. (2017). Gender makes a difference: Investigating consumer purchasing behavior and attitudes toward corporate social responsibility policies. Corporate Social Responsibility and Environmental Management, v. 24, n. 2, 133-144. https://doi.org/10.1002/csr.1401

Kalamas, M.; Cleveland, M.; Laroche, M. (2014). Pro-environmental behaviors for thee but not for me: Green giants, green Gods, and external environmental locus of control. Journal of Business Research, v. 67, n. 2, 12-22. https://doi.org/10.1016/j.jbusres.2013.03.007

Katz-Gerro, T.; Meier Jaeger, M. (2012). Religion, religiosity, and cultural stratification: theoretical links and empirical evidence. En Keister, L. A.; Mccarthy, J.; Finke, R. (Eds.) Religion, Work and Inequality (Research in the Sociology of Work, Vol. 23, pp. 337-366), Emerald Group Publishing Limited. https://doi.org/10.1108/S0277-2833(2012)0000023017

Khan, M. N.; Kirmani, M. D. (2018). Role of religiosity in purchase of green products by Muslim students: Empirical evidences from India. Journal of Islamic Marketing, v. 9, n. 3, 504-526. https://doi.org/10.1108/JIMA-04-2017-0036

Khraim, H. (2010). Measuring Religiosity in Consumer Research from an Islamic Perspective. Journal of Economic and Administrative Sciences, v. 26, n. 1, 52-78. https://doi.org/10.1108/10264116201000003 
Influencia de la religiosidad, la norma subjetiva y la efectividad percibida del consumidor en el consumo socialmente responsable

Kinnear, T. C.; Taylor, J. R.; Ahmed, S. A. (1974). Ecologically concerned consumers: who are they? Ecologically concerned consumers can be identified. Journal of marketing, v. 38, n. 2, 20-24. https://doi.org/10.1177/002224297403800205

Kirmani, M. D.; Khan, M. N. (2016). Environmental attributes and market segmentation: insights from India. International Journal of Management Concepts and Philosophy, v. 9, n. 2, 73-92. https://doi.org/10.1504/IJMCP.2016.077767

Lau, T.C.; Tan, B.C (2009). Religiosity as antecedent of attitude towards green products: An evaluation of religiosity and money ethics. Asean Marketing Journal, v. 1, n. 1, 33-41. https://doi.org/10.21002/amj.v1i1.1979

Leary, R. B.; Minton, E. A.; Mittelstaedt, J. D. (2016). Thou shall not? The influence of religion on beliefs of stewardship and dominion, sustainable behaviors, and marketing systems. Journal of Macromarketing, v. 36, n. 4, 457-470. https://doi.org/10.1177/0276146715626219

Lévy Mangin, J. P.; Varela, J. (2006). Modelización con estructuras de covarianzas en ciencias sociales: temas esenciales, avanzados y aportaciones especiales. Netbiblo.

Lim, E.; Arita, S.; Joung, S. (2019). Advancing Sustainable Consumption in Korea and Japan-From ReOrientation of Consumer Behavior to Civic Actions. Sustainability, v. 11, n. 23, 6683. https://doi.org/10.3390/su11236683

Mandaville, P. (2001) Transnational Muslim Politics: Reimagining the Umma. Editorial Routledge.

Mas'od, A.; Chin, T. A. (2014). Determining socio-demographic, psychographic and religiosity of green hotel consumer in Malaysia. Procedia-social and behavioral sciences, v. 130, 479-489. https://doi.org/10.1016/i.sbspro.2014.04.056

Mayer, F. S.; Frantz, C. M. (2004). The connectedness to nature scale: A measure of individuals' feeling in community with nature. Journal of Environmental Psychology, v. 24, n. 4, 503-515. https://doi.org/10.1016/j.jenvp.2004.10.001

Mazereeuw-van der Duijn Schouten, C.; Graafland, J.; Kaptein, M. (2014). Religiosity, CSR attitudes, and CSR behavior: An empirical study of executives' religiosity and CSR. Journal of business ethics, n. 123, 437-459. https://doi.org/10.1007/s10551-013-1847-3

McAndrew, S.; Voas, D. (2011). Measuring religiosity using surveys. Survey Question Bank. Topic Overview, v. 4, n. 2, 1-15. URL

McDaniel, S. W.; Burnett, J. J. (1990). Consumer religiosity and retail store evaluative criteria. Journal of the Academy of marketing Science, v. 18, n. 2, 101-112. https://doi.org/10.1007/BF02726426 
Millock, K.; Hansen, L. G.; Wier, M.; Anderson, L. M. (junio 2002). Willingness to pay for organic products: A comparison between survey data and panel data from Denmark [Conferencia]. 12th annual EAERE (European Association of Environmental and Resource Economists, Monterey, Estados Unidos. $\underline{\text { URL }}$

Mohamad, Z.F.; Idris, N.; Baharudin, A.; Muhammad, A.; Sulaiman, N. M. N. (2012). The role of religious community in recycling: empirical insight from Malaysia, Journal of Resource, Conservative and Recycling, v. 58, 143-151. https://doi.org/10.1016/j.resconrec.2011.09.020

Mohd Dali, N. R. S.; Yousafzai, S.; Abdul Hamid, H. (2019). Religiosity scale development. Journal of Islamic Marketing, v. 10, n. 1, 227-248. https://doi.org/10.1108/JIMA-11-2016-0087

Mohr, L. A.; Webb, D. J.; Harris, K. E. (2001). Do consumers expect companies to be socially responsible? The impact of corporate social responsibility on buying behavior. Journal of Consumer affairs, v. 35, n. 1, 45-72. https://doi.org/10.1111/j.1745-6606.2001.tb00102.x

Murphy, E. (2014). Manual de guerra espiritual. Grupo Nelson.

Nunnally, J.; Bernstein, I. (1994). Psychometric theory (3a ed.). McGraw-Hill.

Orriols, L. (2013). Social Class, Religiosity, and Vote Choice in Spain, 1979-2008. En Evans, G.; de Graaf, N. D., Political Choice Matters (360-390). Oxford University Press.

Podsakoff, P. M.; Mackenzie, S. B.; Lee, J. Y.; Podsakoff, N. P. (2003). Common method biases in behavioral research: A critical review of the literature and recommended remedies. Journal of Applied Psychology, v. 88, n. 5, 879-903. https://doi.org/10.1037/0021-9010.88.5.879

Prendergast, G. P.; Tsang, A. S. L. (2019). Explaining socially responsible consumption. Journal of Consumer Marketing, v. 36, n. 1, 146-154. https://doi.org/10.1108/JCM-02-2018-2568

Rice, G. (2006). Pro-environmental behavior in Egypt: is there a role for Islamic environmental ethics? Journal of business ethics, v. 65, n. 4, 373-390. https://doi.org/10.1007/s10551-006-0010-9

Ringle, C. M.; Wende, S.; Will, A.; Becker, J. -M. (2018). SmartPLS 3.0 M3 Beta. URL

Rokeach, M. (1969). Value Systems in Religion. Review of Religious Research, v. 11, n. 1, 3-23. https://doi.org/10.2307/3510550

Roshani Perera, C.; Rathnasiri Hewege, C. (2018). Religiosity and environmentally concerned consumer behaviour: 'becoming one with god (nature)' through surrendering environmental identities. International Journal of Consumer Studies, v. 42, n. 6, 627-638. https://doi.org/10.1111/ijcs.12469 
Influencia de la religiosidad, la norma subjetiva y la efectividad percibida del consumidor en el consumo socialmente responsable

Shamdasani, P.; Chon-Lin, G. O.; Richmond, D. (1993). Exploring Green Consumers in an Oriental Culture: Role of Personal and Marketing Mix Factors. En McAlister L.; Rothschild, M. L. (Eds.), NA - Advances in Consumer Research Volume 20 (pp. 488-493). Association for Consumer Research. $\underline{U R L}$

Sharma, C. S.; Sharma, N. (2017). Relationship between consumers' spirituality and green purchasing intentions: The mediation effect of perceived consumer effectiveness. lim Kozhikode Society \& Management Review, v. 6, n. 2, 204-214. https://doi.org/10.1177/2277975216665694

Sharma, N.; Sharma, C. S. (2013). Encouraging green purchasing behavior through green branding. An indian viewpoint. Business Analyst, v. 34, n. 2, 65-76.

Sood, J.; Nasu, Y. (1995). Religiosity and nationality: An exploratory study of their effect on consumer behavior in Japan and the United States. Journal of Business Research, v. 34, n. 1, 1-9. https://doi.org/10.1016/0148-2963(94)00015-7

Straughan, R. D.; Roberts, J. A. (1999). Environmental segmentation alternatives: A look at green consumer behavior in the new millennium. Journal of Consumer Marketing, v. 16, n. 6, 558-575. https://doi.org/10.1108/07363769910297506

Suárez, E.; Hernández, B.; Gil-Giménez, D.; Corral-Verdugo, V. (2020). Determinants of Frugal Behavior: The Influences of Consciousness for Sustainable Consumption, Materialism, and the Consideration of Future Consequences. Frontiers in Psychology, v. 11, 567752. https://doi.org/10.3389/fpsyg.2020.567752

Teimourpour, B.; Heidarzadeh Hanzaee, K. (2011). The impact of culture on luxury consumption behaviour among Iranian consumers. Journal of Islamic Marketing, v. 2, n. 3, 309-328. https://doi.org/10.1108/17590831111164822

Van Raaij, W. F. (1978). Cross-cultural research methodology as a case of construct validity. En Hunt, K.; Abor, A. (Eds.), Advances in Consumer Research (v. 5, pp. 693-701). Association for Consumer Research.

Vela, C.; Ballesteros, C. (2011). La influencia de las creencias religiosas en el consumo. Una aproximación desde las tres religiones del Libro. Icade. Revista de la Facultad de Derecho, n. 8384, 393-411. URL

Vermeir, I.; Verbeke, W. (2006). Sustainable food consumption: Exploring the consumer "attitudebehavioral intention"gap. Journal of Agricultural and Environmental Ethics, v. 19, n. 2, 169-194. https://doi.org/10.1007/s10806-005-5485-3

Webb, D. J.; Mohr, L. A.; Harris, K. E. (2008). A re-examination of socially responsible consumption and its measurement. Journal of business research, v. 61, n. 2, 91-98. https://doi.org/10.1016/i.jbusres.2007.05.007 
Webster Jr, F. E. (1975). Determining the characteristics of the socially conscious consumer. Journal of consumer research, v. 2, n. 3, 188-196. https://doi.org/10.1086/208631

Winkler, J. D.; Kanouse, D. E.; Ware, J. E. (1982). Controlling for acquiescence response set in scale development. Journal of Applied Psychology, v. 67, n. 5, 555-561. https://doi.org/10.1037/0021-9010.67.5.555

Wold, H. (1975). Soft modelling by latent variables: The Non-Linear Iterative Partial Least Squares (NIPALS) approach. Journal of Applied Probability, v. 12, n. S1, 117-142. https://doi.org/10.1017/S0021900200047604

Woodhead, L. (2011). Five concepts of religion. International review of Sociology, v. 21, n. 1, 121-143. https://doi.org/10.1080/03906701.2011.544192 